\title{
Few-Layer Graphene based Printed Flexible Asymmetric Supercapacitor
}

\author{
Ankit Singh $^{\mathrm{a}}$, Kaushik Ghosh ${ }^{\mathrm{b}}$, Sushil Kumar ${ }^{\mathrm{b}}$, Ashwini K.Agarwal ${ }^{\mathrm{c}}$, Manjeet Jassal ${ }^{\mathrm{c}}$, \\ Pranab Goswami ${ }^{\mathrm{a}}$, Harsh Chaturvedi ${ }^{\mathrm{a}}$ * \\ ${ }^{a}$ Centre for Energy, Indian Institute of Technology, Guwahati, Assam,781039, India \\ ${ }^{b}$ Institute of Nano Science and Technology, Mohali, Punjab, 160062, India \\ ${ }^{c}$ SMITA research lab, Indian Institute of Technology, Delhi, New Delhi, 110016, India \\ (*harshc@iitg.ac.in)
}

\begin{abstract}
We have developed a flexible planar asymmetric supercapacitor demonstrating high energy storage capability which can be utilized to power various flexible and wearable electronic devices. Locally available cheap source of biomass such as banana peel was utilized for synthesising carbonaceous materials like few-layer graphene and activated carbon for electrode material application. Few-layer graphene was synthesized by heating banana peel at high temperature under inert atmosphere followed by crushing with mortar-pestle. Activated carbon was synthesized by heating banana peel impregnated with $\mathrm{KOH}$ at high temperature in absence of air. The device was fabricated by using low cost screen printing technique to print current collectors followed by deposition of active electrode materials and sandwiching filter paper soaked in gel electrolyte in between the electrodes. The fabricated devices showed high areal capacitance of $88.31 \mathrm{mF} / \mathrm{cm}^{2}$ at $10 \mathrm{mV} / \mathrm{s}$ scan rate. The device also showed satisfactory performance under multiple electronic cycling (100 cycles) and bending conditions. The device can be fabricated on large scale using low cost screen printing technique and cheaply available biomass which can be further utilized for developing emerging flexible electronics
\end{abstract}

Keywords: flexible, planar, asymmetric supercapacitor, high areal capacitance, few layer graphene, activated carbon, screen printing.

\section{Introduction}

Recently the demand for wearable electronics has increased considerably and is expected to grow further in the future. However, the practical application is limited due to lack of wearable energy sources. Lithium based batteries are predominantly used in modern-day electronics but it has limitations like threat of explosion or electrolyte leakage which makes it 
unfit for wearable application. Due to this, a need for safe, environment friendly, low cost, thin and mechanically flexible energy storing device has arisen which would store/supply energy to wearable devices. Wearable energy storage devices represent a significant paradigm shift in consumer electronics since they eliminate the necessity for carriage of bulky devices. Hybrid supercapacitor is a promising alternative for powering wearable electronics especially sensors. This hybrid supercapacitor has advantages of EDLC and psuedocapacitance.[1,2] We are fabricating carbon based thin mechanically flexible planar asymmetric supercapacitor using screen printing technique for printing current collector and incorporating synthesized few-layer graphene and activated carbon from banana peel and also transition metal oxide as the electrode material. This flexible supercapacitor can be incorporated into wearable electronics in the domain of biomedical, smart textile, computing and processing. They can also be utilized for environmental parameter testing and monitoring applications.[3]

\section{Experimental}

\section{Synthesis of activated carbon}

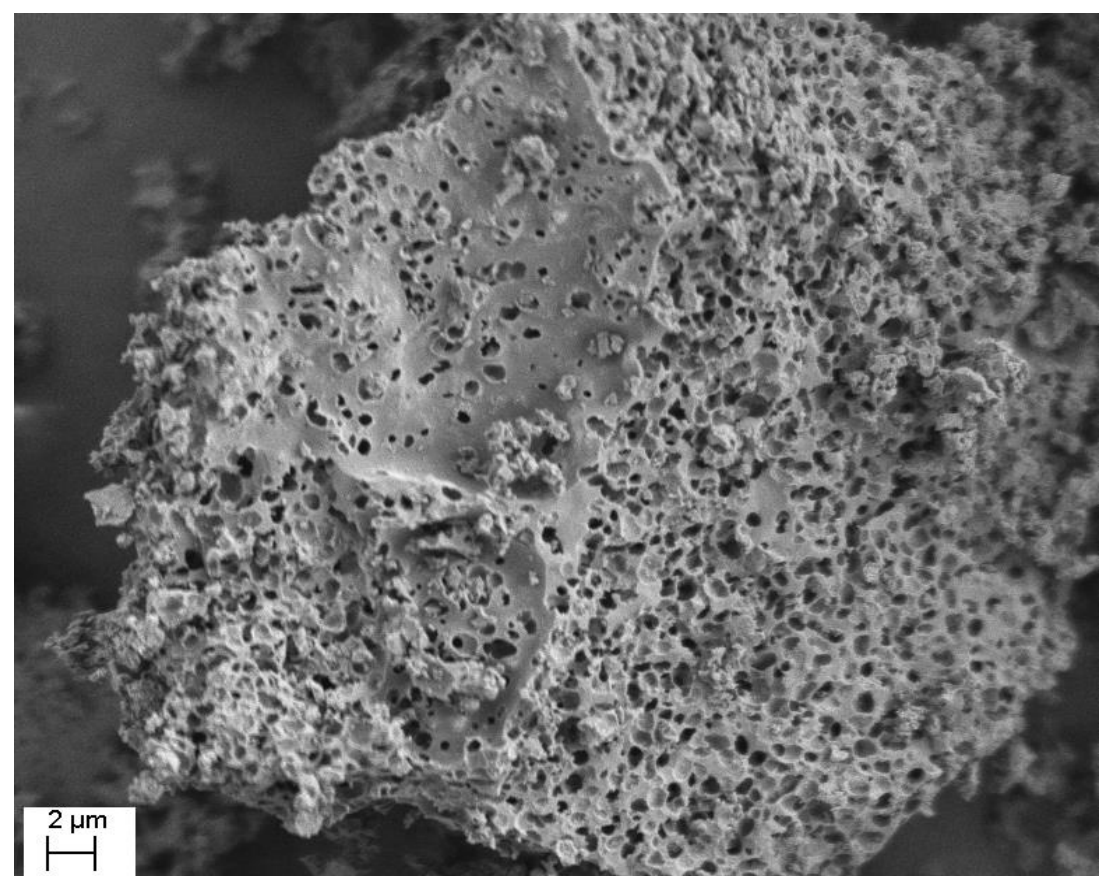

Figure 1: FESEM image of activated carbon at $5 \mathrm{kX}$ magnification showing well porous structure

Locally available cheap biomass such as banana peel (musa acuminata) was used as a precursor for synthesizing activated carbon. The peel was washed several times with distilled water and left overnight in vacuum oven at $80^{\circ} \mathrm{C}$ to remove excess water. Small quantity of $\mathrm{KOH}$ flakes $(3 \mathrm{~g})$ were mixed with DI water $(50 \mathrm{ml})$ to prepare aqueous solution of $\mathrm{KOH}$. The 
dried peel was added to this $\mathrm{KOH}$ solution in small amount $(3 \mathrm{~g})$ and was under constant magnetic stirring at $200 \mathrm{rpm}$ for 2 hours. The peel was then taken out from the solution and left overnight in vacuum oven at $80^{\circ} \mathrm{C}$ to remove excess water. The peel impregnated with $\mathrm{KOH}$ was heated at $600{ }^{\circ} \mathrm{C}$ for $1 \mathrm{hr}$ in a muffle furnace in absence of air. The resulting carbon material was washed multiple times with dilute HCL and distilled water until the $\mathrm{pH}$ reached neutral condition $(\mathrm{pH} 7)$ and was heated in vacuum oven at $80^{\circ} \mathrm{C}$ overnight to remove excess water resulting in activated carbon. The synthesized activated carbon was investigated using Brunauer-Emmett-Teller (BET) surface area analyser and field emission scanning electron microscopy (FESEM). From the FESEM image (figure 1), well porous structure of the synthesized activated carbon is observed with pore size of few hundred nanometer. The activated carbon has BET surface area of $62.03 \mathrm{~m}^{2} / \mathrm{g}$. [4]

\section{Synthesis of few-layer graphene}
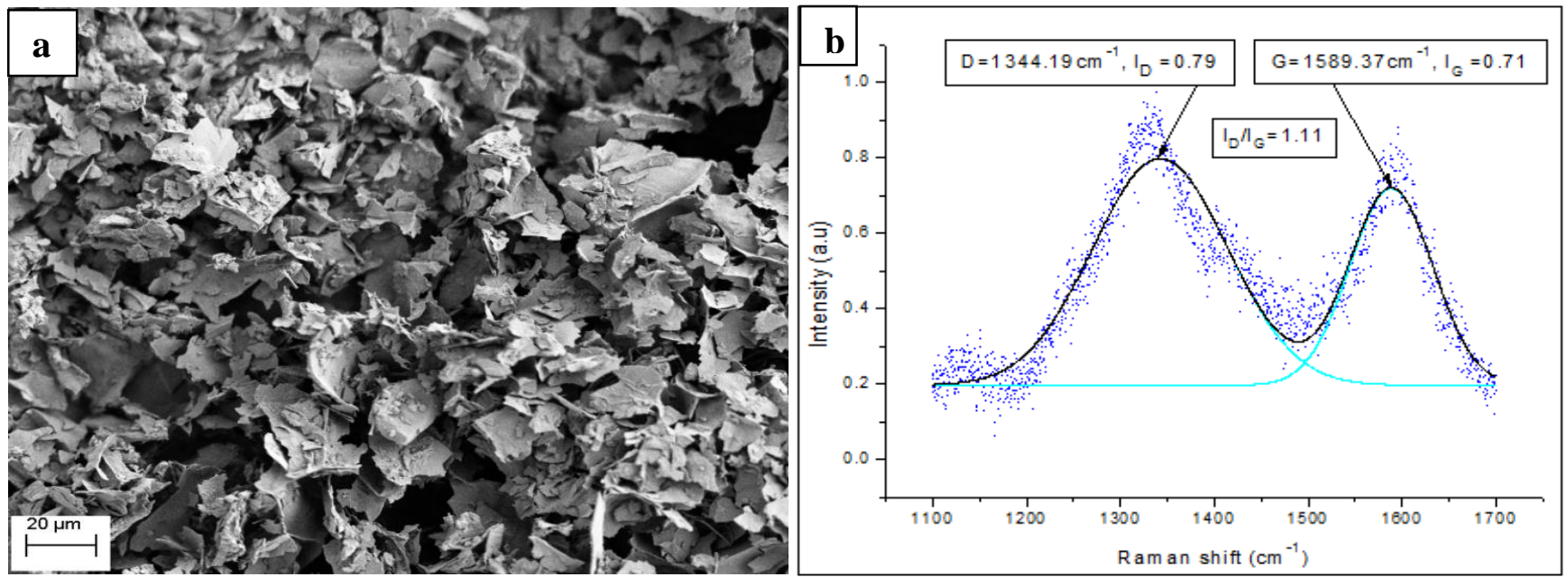

Figure 2: (a) FESEM image of few-layer graphene at 1kX magnification, (b) Raman spectrum of few-layer graphene at $633 \mathrm{~nm}$.

Banana peel was also used for synthesizing few-layer graphene. Thoroughly washed and dried peel was washed with ethanol and acetone and was dried at room temperature. The peel was then heated in a tubular furnace at $950^{\circ} \mathrm{C}$ for $2 \mathrm{hrs}$ under argon gas environment. The carbon residue was crushed in mortar-pestle resulting in few-layer graphene. The few-layer graphene was investigated using Raman spectroscopy and FESEM. Raman spectrum (Figure 2(b)) verified the presence of few-layer graphene and its small size was observed using FESEM image (Figure 2(a)). [5] 


\section{Fabrication of planar asymmetric supercapacitor}

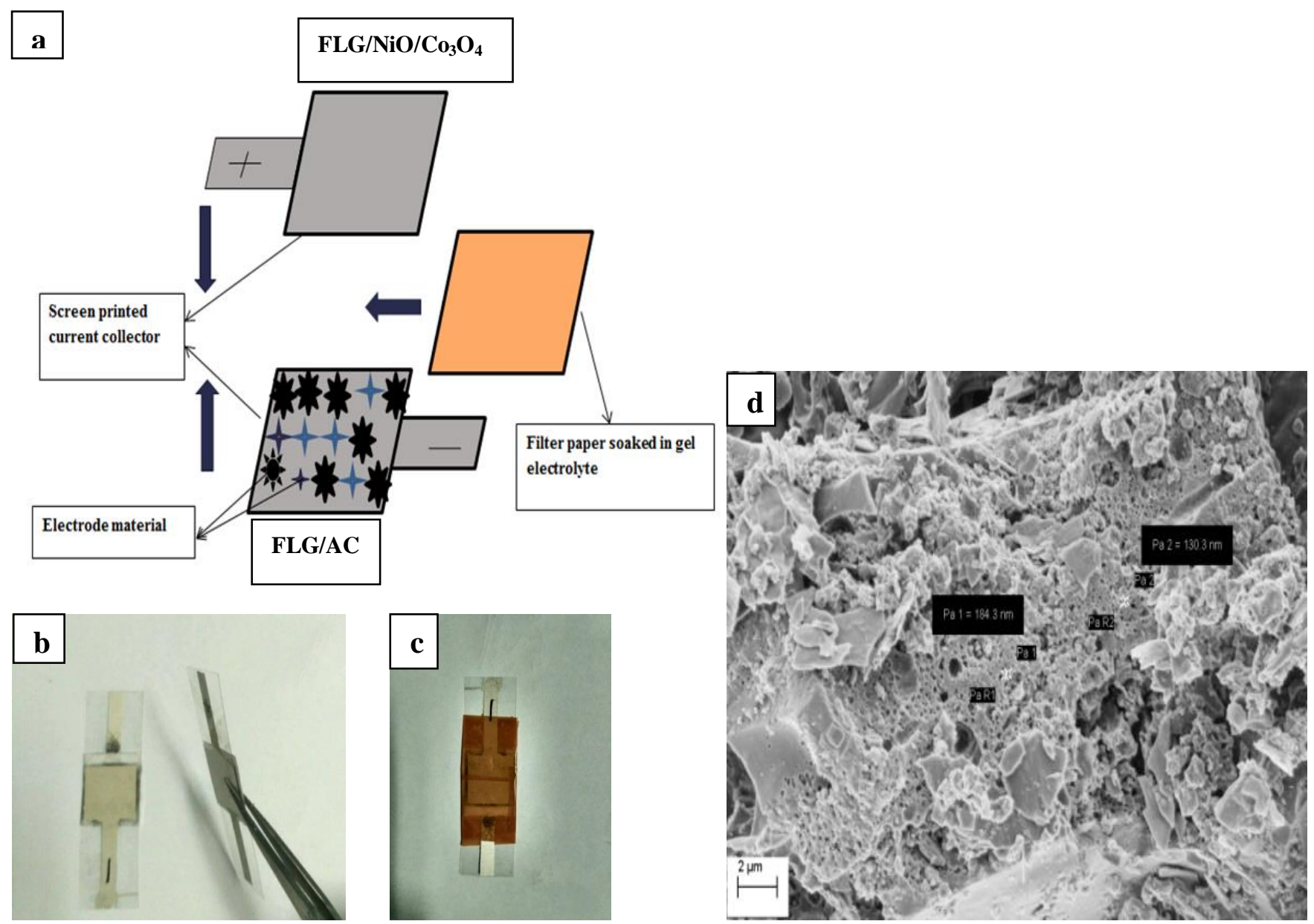

Figure 3: (a) Schematic description of fabrication of planar asymmetric supercapacitor, (b) Optical image of fabricated supercapacitor, (c) planar supercapacitor covered with sticky tape, (d) FESEM of FLG/AC at 10kX magnification

In order to fabricate planar asymmetric supercapacitor, current collectors were printed by screen printing conducting silver ink on flexible PET substrate. Equal amount (1mg) of fewlayer graphene (FLG) and activated carbon (AC) were sprinkled on one of the printed current collector forming the anode. Equal amount $(0.5 \mathrm{mg})$ of $\mathrm{NiO} / \mathrm{Co}_{3} \mathrm{O}_{4}$ along with $1 \mathrm{mg}$ of fewlayer graphene was sprinkled on the other printed current collector forming the cathode. [6] $\mathrm{PVA} / \mathrm{H}_{3} \mathrm{PO}_{4}$ gel electrolyte was prepared by mixing PVA polymer in DI water (1: 10 by \%wt) under constant stirring at $90^{\circ} \mathrm{C}$ followed by addition of $1 \mathrm{ml}$ of $\mathrm{H}_{3} \mathrm{PO}_{4}$ acid.[7] Filter paper soaked in gel electrolyte was sandwiched between the two asymmetric electrodes as shown in figure 3(a). The front and side view of fabricated supercapacitor is shown in the figure 3(b),(c). Anode electrode material (FLG/AC) was observed by FESEM, showing pore size of activated carbon to be in range of 100-200 nm and few-layer graphene flakes. 


\section{Device characterization}
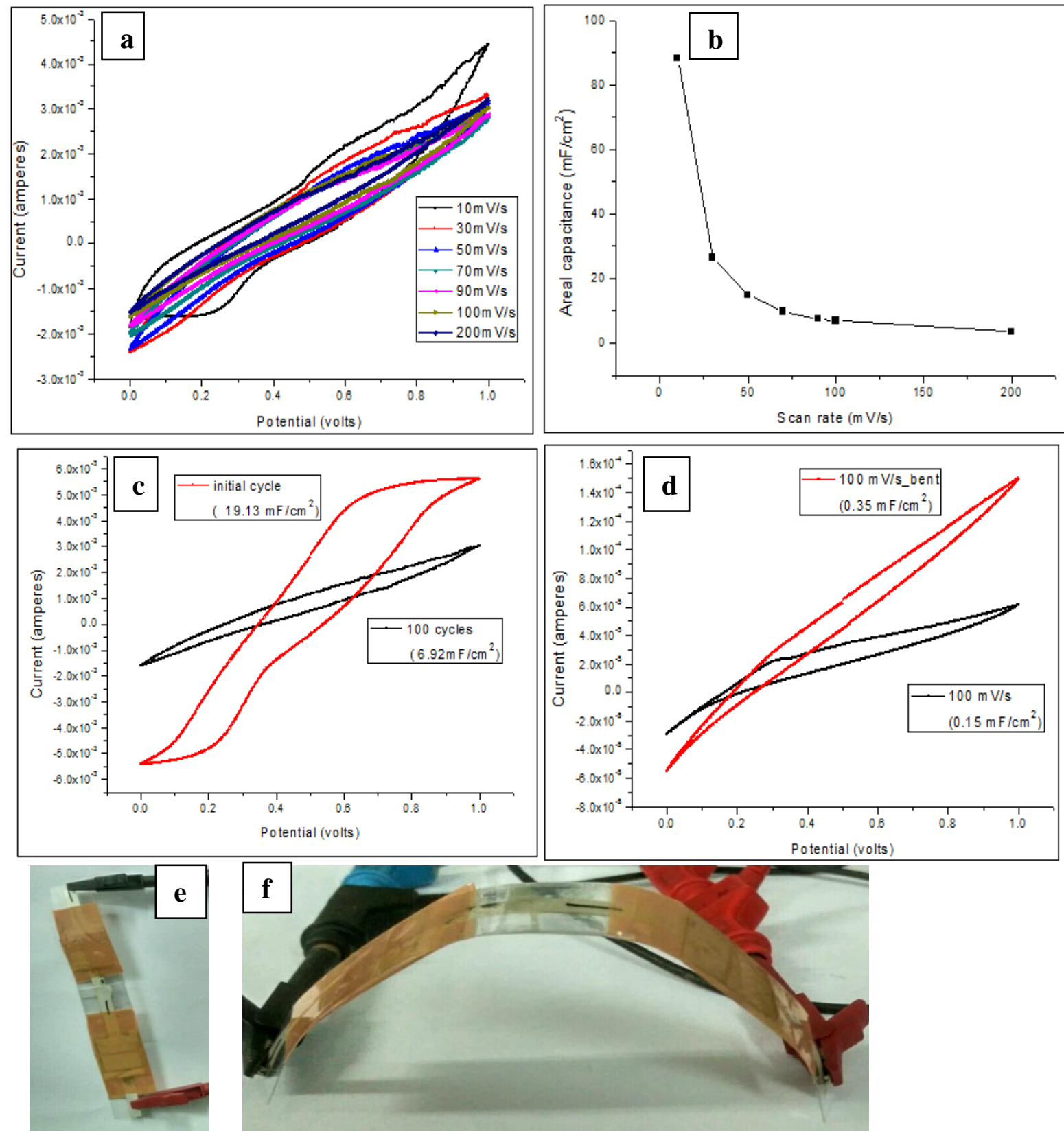

Figure 4: (a) CV profile of supercapacitor at different scan rates, (b) Areal capacitance plot at different scan rates, (c) About $36 \%$ of capacitance was retained after $\sim 100 \mathrm{CV}$ cycles at 100mV/s scan rate, (d) Increase of $133 \%$ in capacitance is observed during bend test of series connected supercapacitor at $100 \mathrm{mV} / \mathrm{s}$, Series connected supercapacitor in (e) unbent and (f) bent conditions.

The fabricated supercapacitor was tested by cyclic voltammetry $(\mathrm{CV})$ at scan rates ranging from $10 \mathrm{mV} / \mathrm{s}$ to $200 \mathrm{mV} / \mathrm{s}$ (Figure 4(a)). Corresponding areal capacitance was calculated by formula, $C s=\frac{A}{a * v * V} \quad$ where, $\mathrm{A}$ is area of $\mathrm{CV}$ curve, a= area of active part of device $(3.61$ $\mathrm{cm}^{2}$ ), $\mathrm{v}$ is scan rate in $\mathrm{mV} / \mathrm{s}$ and $\mathrm{V}$ is potential window (1V) (Figure 4(b)). [8] Lifecycle test was done by carrying out $100 \mathrm{CV}$ cycles at $100 \mathrm{mV} / \mathrm{s}$ scan rate and the supercapacitor 
retained $36 \%$ of capacitance (Figure 4(c)). Series connected planar supercapacitor were tested under unbent and bent condition and an increase in the capacitance (133\%) was observed under bent condition which may be due to better interaction between electrode and electrolyte (Figure 4(d),(e),(f)).

\section{Conclusion}

The planar asymmetric supercapacitor was fabricated by incorporating synthesized fewlayer graphene and activated carbon from cheaply available biomass (banana peel) along with transition metal oxide $\left(\mathrm{NiO} / \mathrm{Co}_{3} \mathrm{O}_{4}\right)$ as electrode materials. Also, low cost screen printing technique was used to print current collectors. Few-layer graphene has lower resistance and therefore enhanced charge distribution improving the performance of the device. Porous activated carbon provided large surface area for charge storage therefore enhancing the capacitance. Planar asymmetric supercapacitor demonstrated high areal capacitance $\left(88.31 \mathrm{mF} / \mathrm{cm}^{2}\right)$ at $10 \mathrm{mV} / \mathrm{s}$ scan rate due to presence of transition metal oxide as electrode material. The fabricated device demonstrated satisfactory performance under multiple cycling and bending conditions. Different combination of electrode materials should be explored to get desirable cyclability and higher capacitance. Hence, fabricated supercapacitor demonstrating significantly high energy storing capacity can be utilized for developing emerging flexible electronics which can be further incorporated with wearable devices.

\section{Acknowledgement}

We would like to thank Centre for Energy, Central Instruments Facility of Indian Institute Of Technology, Guwahati. We would also like to thank G Labs Pvt ltd, Kolkata for their support.

\section{References}

[1] US 2800616, Becker, H.I., "Low voltage electrolytic capacitor", issued 1957-07-23

[2]B.E.Conway. Transition from Supercapacitor to Battery Behaviour in Electrochemical Energy storage. J.Electrochem.Soc.138(6): 1539-1548.

[3] X. Li and B. wei. Supercapacitors based on nanostructured carbon. Nano Energy, 2, 2, (2013), 159-173. 
[4]Wang, J. Kaskel, Stefan. KOH activation of carbon-based materials for energy storage. J. Mater. Chem., 22, (2012), 23710-23725.

[5] T. Purkait, G. Singh, M. Singh et al. Large area few-layer graphene with scalable preparation from waste biomass for high-performance supercapacitor. Scientific Reports, 7, (2017), 15239.

[6] Y. Wang, J. Guo, T. Wang. Mesoporous Transition Metal Oxides for Supercapacitors. Nanomaterials, 5, (2015), 1667-1689.

[7] Chen, Q. Li, X. Zang et al. Effect of different gel electrolytes on graphene-based solid-state supercapacitors. RSC Adv, 4, 68, (2014), 36253-36256.

[8] B.D. Boruah, A. Maji, A. Misra. . Flexible Array of Microsupercapacitor for Additive Energy Storage Performance Over a Large Area. ACS Appl. Mater. Interfaces, 10, (2018), $15864-15872$. 